Check for updates

Cite this: RSC Adv., 2017, 7, 53301

Received 3rd October 2017

Accepted 31st October 2017

DOI: $10.1039 / c 7 r a 10908 c$

rsc.li/rsc-advances

\section{Surface modification of anhydrite whiskers and their potential application for durable superhydrophobic coatings}

\author{
Tianzeng Hong, ${ }^{\text {abc }}$ Yabin Wang, ${ }^{\text {a }}$ Xueying Nai, (D) *a Yaping Dong, ${ }^{\text {*a }}$ Xin Liu ${ }^{\mathrm{c}}$ \\ and $\mathrm{Wu} \mathrm{Li}^{\mathrm{ac}}$
}

\begin{abstract}
Durability issues have been an obstacle for superhydrophobic coating applications for a long time. Here, we report the modification of anhydrite whiskers that can be used for the fabrication of superhydrophobic coatings with high durability. Anhydrite whiskers with core/shell structures are prepared via hydrolysis and condensation processes of tetraethyl orthosilicate (TEOS) followed by silane modification. Modified anhydrite whiskers were examined by field emission scanning electron microscopy (FESEM), transmission electron microscopy (TEM), Fourier transform infrared (FT-IR) spectroscopy, X-ray powder diffraction (XRD) and X-ray photoelectron spectroscopy (XPS). The results indicate that anhydrite whiskers exhibit an obviously hydrophobic quality after modification, reaching a contact angle of up to $123^{\circ}$. The thickness of the surface modification layer is $10-20 \mathrm{~nm}$. The modified anhydrite whiskers were used to fabricate a superhydrophobic coating. Modified anhydrite whiskers create a rough structure on the glass substrate surface. This roughness develops a Cassie-Baxter regime, enhancing the superhydrophobicity of the coating. The water contact angle of the coating is above $150^{\circ}$. This method is expected to apply to similar materials for the fabrication of durable superhydrophobic coatings.
\end{abstract}

\section{Introduction}

Superhydrophobic surfaces are eagerly anticipated for use in applications such as self-cleaning coatings, ${ }^{1-5}$ non-wetting fabrics, ${ }^{6,7}$ and anti-icing and anti-fogging surfaces. ${ }^{8}$ A superhydrophobic coating is usually fabricated by coating with a layer of low surface energy materials on the substrate surface constructed with a similar surface morphology to that of lotus leaves. However, a superhydrophobic coating can easily lose its superhydrophobic properties due to wear. ${ }^{9-11}$ It is of great significance for practical applications to carry out research on enhancing the wear robustness of superhydrophobic coatings.

The calcium sulfate whisker is a typical high strength, energy-saving, low cost and environmentally friendly material widely applied as a filler in composites. ${ }^{\mathbf{1 2 - 1 5}}$ The calcium sulfate whisker exists as three types: dehydrate, hemihydrate, and anhydrite. Among the three types, the anhydrite whisker has the best physical and chemical performance. Compared with the dehydrate whisker and the hemihydrate whisker, the anhydrite

\footnotetext{
${ }^{a}$ Key Laboratory of Salt Lake Resources Chemistry, Qinghai Institute of Salt Lakes, Chinese Academy of Sciences, Xining 810008, China. E-mail: naixy@isl.ac.cn; dyp811@isl.ac.cn

${ }^{b}$ Key Laboratory of Comprehensive and Highly Efficient Utilization of Salt Lake Resources, Qinghai Institute of Salt Lakes, Chinese Academy of Sciences, Xining 810008, China

'University of Chinese Academy of Sciences, Beijing 100049, China
}

whisker has higher thermal and chemical stability. In the past few decades, whiskers have been applied widely as fillers in the areas of plastics, ${ }^{16}$ rubbers, ${ }^{17,18}$ paper making, ${ }^{19}$ ceramics, ${ }^{20}$ etc. However, whiskers have not been used for designing superhydrophobic coatings. In fact, the calcium sulfate whisker is a promising building material for the design of superhydrophobic coatings. In our study, anhydrite whiskers were modified by hydrolysis and condensation with tetraethyl orthosilicate (TEOS). The anhydrite whisker surface was coated with a -Si-O-Si- network after being modified with TEOS, and the resulting surface was modified with octadecyl trichlorosilane (OTS). Modified anhydrite whiskers showed good hydrophobic character and low surface energy. The superhydrophobic coating was fabricated via a facile approach using hydrophobic anhydrite whiskers. This method could be a potential approach for the large-scale production of durable superhydrophobic coatings with fibrous materials.

\section{Experimental}

\subsection{Materials}

All chemicals in the present work were used as received without further purification. The $\mathrm{CaSO}_{4} \cdot 2 \mathrm{H}_{2} \mathrm{O}$ was supplied by Sinopharm Chemical Reagent Co., Ltd.; absolute ethanol was purchased from Tianjin chemical reagent plant; ammonia $\left(\mathrm{NH}_{3}, 25 \%\right.$ in water, A.R.), tetraethyl orthosilicate (TEOS), octadecyl trichlorosilane (OTS), cyclohexane and methylene 
blue were obtained from Aladdin Industrial Corporation. The glass paint (K2O8-SO100), thinner (830-0006) and hardener (205-1100) were purchased from JiangMen Pule Chemical Co. Ltd.

\subsection{Surface modification of anhydrite whiskers}

The synthesis of anhydrite whiskers was carried out as reported in our previous work. ${ }^{21} 2 \mathrm{~g}$ of anhydrite whiskers was dispersed in $80 \mathrm{~mL}$ absolute ethanol and $20 \mathrm{~mL}$ distilled water under ultrasonication for $2 \mathrm{~min}$. Then, $1.5 \mathrm{~mL}$ ammonium hydroxide and $1 \mathrm{~mL}$ TEOS were added into the as-prepared dispersion and stirred for $10 \mathrm{~h}$. The slurry was filtered off and washed with distilled water. The product was dispersed in $100 \mathrm{~mL}$ cyclohexane, and $0.06 \mathrm{~g}$ OTS was added into the slurry of anhydrite whiskers and stirred for $3 \mathrm{~h}$. The anhydrite whisker suspension was filtered and washed with cyclohexane to remove excessive OTS. The final sample was then dried at $100{ }^{\circ} \mathrm{C}$ for $1 \mathrm{~h}$.

\subsection{Superhydrophobic coating fabrication}

In order to know if the modified anhydrite whiskers could be potentially used in practical superhydrophobic coatings, commercial paint was chosen as an experimental material.

$0.5 \mathrm{~g}$ glass paint, $10 \mathrm{~g}$ thinner and $0.2 \mathrm{~g}$ hardener were mixed into a homogeneous solution and centrifuged to obtain the supernatant. $0.5 \mathrm{~g}$ modified anhydrite whiskers and $0.03 \mathrm{~g}$ OTS were added into the supernatant, and they were subjected to ultrasonication for $10 \mathrm{~min}$. Then, some of the obtained suspension was dropped onto a glass substrate. The slurry flowed over the substrate and evenly coated the glass substrate. The obtained glass substrate was dried and a superhydrophobic coating was fabricated on the glass substrate.

\subsection{Characterization}

The water contact angle was measured with instrument DSA30, Kruss, Germany by dropping deionized water $(3 \mu \mathrm{L})$ on the coating surface on five different spots. The average of five measurements was used as the contact angle result. Characterization of the morphology of the anhydrite whiskers was observed using a field emission scanning electron microscope (FESEM, SU8010, Japan). TEM images were obtained on a scanning transmission electron microscope (TEM, JEM-2100, Japan). The crystal structures of the anhydrite whiskers were determined by X-ray powder diffraction (XRD, X'PRO Pert, Netherlands). The surface functional groups and chemical composition of the sample were characterized using Fourier transform infrared spectrophotometry (FT-IR, NEXUS, USA) and X-ray photoelectron spectroscopy (XPS, Thermo escalab 250Xi, USA), respectively.

\section{Results and discussion}

\subsection{Characterization of the anhydrite whiskers}

The morphology of the anhydrite whiskers is shown in the SEM images (Fig. 1a). The surface of the anhydrite whiskers is smooth. The inset in Fig. 1a shows that the water droplet can quickly infiltrate due to the hydrophilicity of the anhydrite

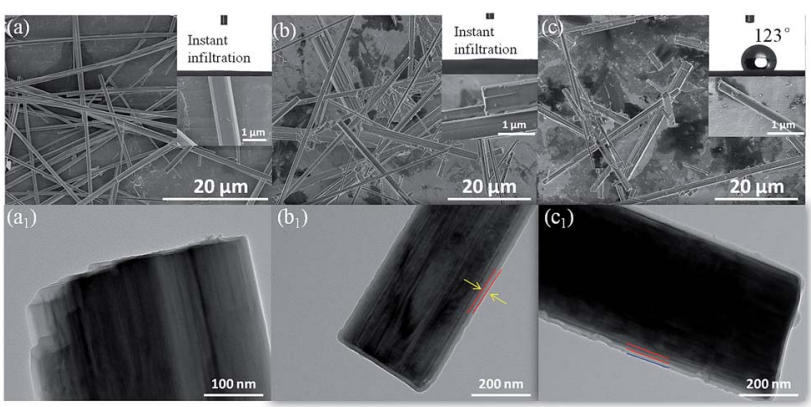

Fig. 1 FESEM images of (a) the anhydrite whiskers, (b) the TEOS modified anhydrite whiskers and (c) the OTS modified anhydrite whiskers; TEM images of $\left(a_{1}\right)$ an anhydrite whisker, $\left(b_{1}\right)$ a TEOS modified anhydrite whisker and $\left(c_{1}\right)$ an OTS modified anhydrite whisker.

whiskers. The static contact angle for the anhydrite whisker surface is $0^{\circ}$. Fig. $1 \mathrm{~b}$ shows that the surface of the anhydrite whiskers is coarse after TEOS modification. An amorphous layer covered the surface of the anhydrite whiskers. The thickness of the coating layer is approximately $15 \mathrm{~nm}$ (Fig. $1 \mathrm{~b}_{1}$ ), and the hydrophilicity of the anhydrite whiskers has not changed (Fig. 1b). To obtain a hydrophobic anhydrite whisker, the TEOS modified anhydrite whisker is chemically modified with longchain OTS. No evident transformation can be observed from the SEM images (Fig. 1(b and c)). The anhydrite whisker surface character is changed from hydrophilic to hydrophobic. The TEM results demonstrate that the thickness of the OTS nanofilm is approximately $16 \mathrm{~nm}$ (Fig. 1c $\mathrm{c}_{1}$ ).

The XRD patterns of the anhydrite whiskers before and after modification show identical diffraction peaks at approximately $2 \theta=25^{\circ}, 31^{\circ}, 41^{\circ}, 48^{\circ}$, and $56^{\circ}$, assigned to (020), (210), (212), (230), and (232), respectively (Fig. 2). The diffraction peaks of the modified anhydrite whiskers remained unchanged (Fig. 2(b and c)), indicating that modification reactions occur on the surface of the anhydrite whiskers only. No characteristic diffraction peaks of $\mathrm{SiO}_{2}$ are observed because of its lower loading content and weak crystallization. On the other hand, it also implies a uniform coating of the modifier on the anhydrite whisker surface. By comparing the patterns, the intensity of the characteristic peaks of TEOS modified anhydrite whiskers and

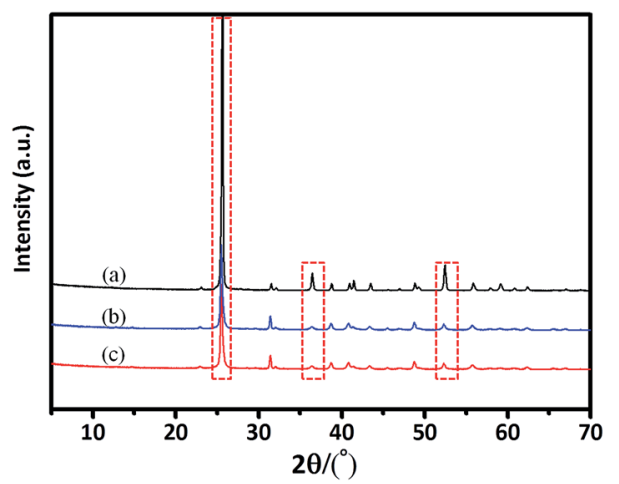

Fig. 2 XRD patterns of the (a) anhydrite whisker, (b) TEOS modified anhydrite whisker and (c) OTS modified anhydrite whisker. 
OTS modified anhydrite whiskers becomes weaker than that of the bare anhydrite whiskers, which may be attributed to the presence of amorphous $\mathrm{SiO}_{2}$ and long-chain saline successfully coated onto the surfaces of the anhydrite whiskers ${ }^{22,23}$ (Fig. 2).

Fig. 3 shows the FT-IR spectra of the anhydrite whisker, TEOS modified anhydrite whisker and OTS modified anhydrite whisker. The intense band at approximately $1156 \mathrm{~cm}^{-1}$ is ascribed to the stretching vibration of $\mathrm{S}-\mathrm{O}^{24}$ (Fig. 3(a-c)). As shown in Fig. 3(b and c), several bands appeared in the spectra of the TEOS modified anhydrite whisker and OTS modified anhydrite whisker, compared with that of the anhydrite whisker. The band at $954 \mathrm{~cm}^{-1}$ could be ascribed to the bending vibration of $\mathrm{Si}-\mathrm{OH}$, which suggests that TEOS is hydrolyzed and adsorbed on the anhydrite whisker surface. In addition, the bands at $456 \mathrm{~cm}^{-1}, 795 \mathrm{~cm}^{-1}$ and $1090 \mathrm{~cm}^{-1}$ are respectively attributed to the symmetric and asymmetric stretching vibrations of $\mathrm{Si}-\mathrm{O}-\mathrm{Si}^{25,26}$ The bands at $2800-3000 \mathrm{~cm}^{-1}$ can be assigned to the symmetric and asymmetric stretching vibrations of $-\mathrm{CH}_{2}-$ groups ${ }^{27,28}$ (Fig. 3c), confirming the existence of OTS on the anhydrite whisker surface. Compared with Fig. 3c, there are no $-\mathrm{CH}_{2}-$ vibration bands in Fig. $3 \mathrm{~b}$ indicating that TEOS is hydrolyzed completely.

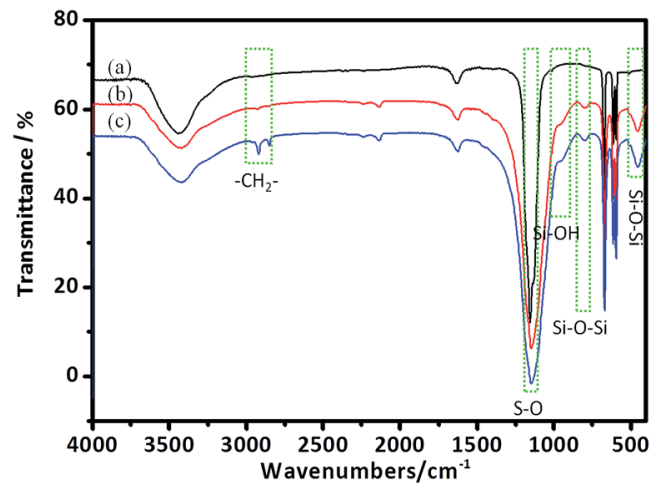

Fig. 3 FT-IR spectra of the (a) anhydrite whisker, (b) TEOS modified anhydrite whisker and (c) OTS modified anhydrite whisker.

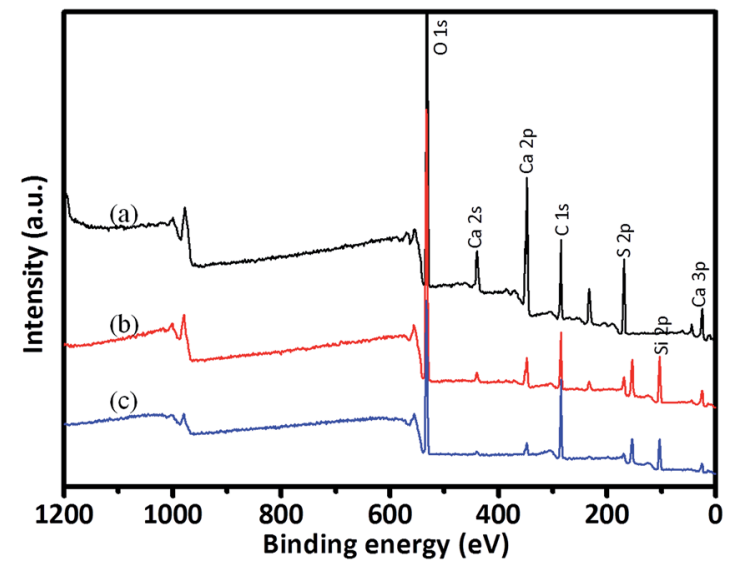

Fig. 4 XPS spectra of the (a) anhydrite whisker, (b) TEOS modified anhydrite whisker and (c) OTS modified anhydrite whisker.
Table 1 The elemental content of the anhydrite whisker, TEOS modified anhydrite whisker and OTS modified anhydrite whisker surface

\begin{tabular}{llcllll}
\hline & $\mathrm{Ca} / \%$ & $\mathrm{~S} / \%$ & $\mathrm{O} / \%$ & $\mathrm{C} / \%$ & $\mathrm{Si} / \%$ & $\mathrm{O} / \mathrm{Si}$ \\
\hline Anhydrite whisker & 11.41 & 12.72 & 49.57 & 26.3 & - & - \\
TEOS modified & 3 & 4.16 & 50.28 & 23.53 & 19.03 & 2.64 \\
OTS modified & 1.54 & 2.6 & 37.89 & 40.31 & 17.65 & 2.15 \\
\hline
\end{tabular}

The XPS full scan spectra of the anhydrite whisker, TEOS modified anhydrite whisker and OTS modified anhydrite whisker are shown in Fig. 4. All three samples show several main characteristic peaks located at $169 \mathrm{eV}, 284 \mathrm{eV}, 347 \mathrm{eV}$ and $531 \mathrm{eV}$, which are respectively attributed to the S 2p, C 1s, Ca 2p and $\mathrm{O} 1 \mathrm{~s}$ signals. For the TEOS modified anhydrite whiskers and the OTS modified anhydrite whiskers, aside from the above four types of element, a Si 2p signal at $103 \mathrm{eV}$ is also seen (Fig. 4(b and c)). The signal intensities of S 2p, Ca 2p and $\mathrm{O} 1 \mathrm{~s}$ are weaker than those of the bare anhydrite whisker, which can be attributed to the thickness of the coating increasing (Fig. 4(b and c)).

Table 1 shows the elemental content of the anhydrite whisker surfaces. Compared with the raw anhydrite whisker, the TEOS modified anhydrite whisker contains $\mathrm{Si}$ (19.03 wt\%) and the $\mathrm{O} / \mathrm{Si}$ ratio is 2.64 (lower than 4), demonstrating that TEOS is hydrolyzed and then partly polymerized on the surface of the anhydrite whisker. After OTS modification, the $\mathrm{O} / \mathrm{Si}$ ratio decreased to 2.15 and the content of carbon increased significantly (nearly doubled), indicating that OTS exists on the anhydrite whisker surface.

High-resolution spectra of $\mathrm{Ca} 2 \mathrm{p}, \mathrm{S} 2 \mathrm{p}, \mathrm{O} 1 \mathrm{~s}$ and Si $2 \mathrm{p}$ are shown in Fig. 5. The FT-IR results indicate that there are $\mathrm{Si}-\mathrm{OH}$ groups on the anhydrite whisker surface. $\mathrm{Si}-\mathrm{OH}$ may be adsorbed through hydrogen bond interactions with $\mathrm{SO}_{4}{ }^{2-}$ on the anhydrite whisker surface. The interaction of the hydrogen
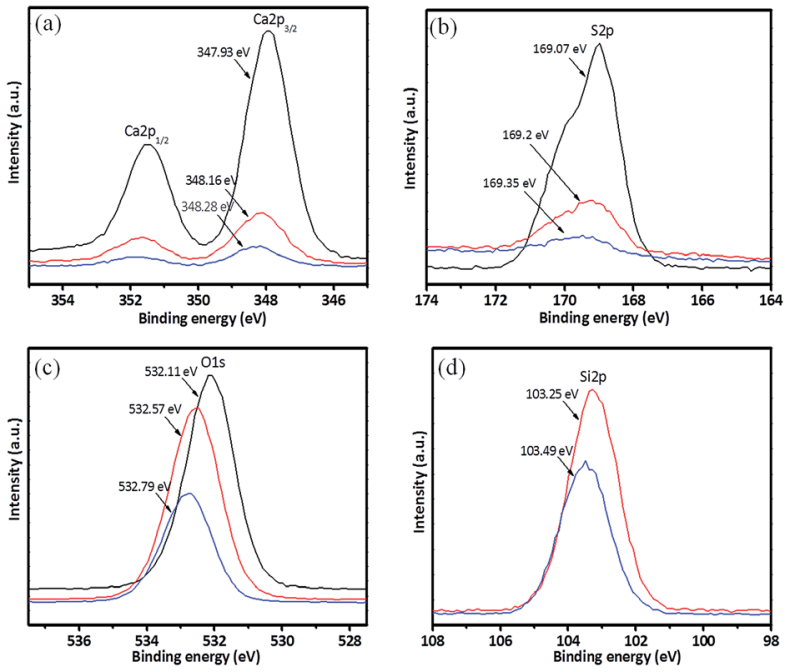

Fig. 5 High-resolution spectra of Ca 2p (a), S 2p (b), O 1s (c) and Si 2p (d) peaks: anhydrite whisker (black line), TEOS modified anhydrite whisker (red line) and OTS modified anhydrite whisker (blue line). 


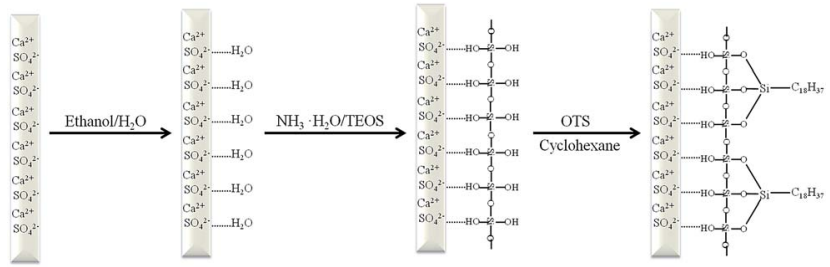

Fig. 6 The surface modification mechanism of the anhydrite whisker.

bonds leads to a decrease in the electron density of $\mathrm{O}$ in $\mathrm{SO}_{4}{ }^{2-}$ and leaves $\mathrm{S}$ with a high positive charge, which causes the binding energy of S $2 p$ to increase slightly due to a lower electron density (Fig. 5(a and b)). Similarly, the binding energy of Ca $2 p$ also increases. Compared with the raw anhydrite whiskers, the binding energy of $\mathrm{O} 1 \mathrm{~s}$ increases after surface modification, demonstrating that the chemical state of $\mathrm{O}$ on the anhydrite whisker surface changes from $\mathrm{SO}_{4}{ }^{2-}$ to $\mathrm{Si}-\mathrm{O}-\mathrm{Si}^{29,30}$ (Fig. 5c). After modification with OTS, the $\mathrm{O} / \mathrm{Si}$ ratio shows a decrease of 0.49 due to the OTS reacting with silanol groups on the anhydrite whisker surface. The increased bridging oxygen leads to a decrease in the bond length of $\mathrm{Si}-\mathrm{O}$ and to a decrease in the electron density in the nucleus. The binding energies of $\mathrm{Si} 2 \mathrm{p}$ and $\mathrm{O} 1 \mathrm{~s}$ both increased ${ }^{31-33}$ (Fig. 5(c and d)).

Fig. 6 shows the possible mechanism of the anhydrite whisker surface modification. A large number of water molecules is adsorbed on the anhydrite whisker surface due to hydrogen bond interactions. TEOS is hydrolyzed and polymerized on the surface of the anhydrite whisker. There are many silanol groups on the anhydrite whisker surface. Then OTS reacts with the silanol groups and further polymerizes on the anhydrite whisker surface. ${ }^{34}$ The Si-OH groups in the interior of the coating are adsorbed on the anhydrite whisker surface by hydrogen bond interactions. The modified anhydrite whisker is not only hydrophobic but also highly stable. This modification can greatly enhance the durability of superhydrophobic coatings. ${ }^{35,36}$

\subsection{The superhydrophobic coating}

Modified anhydrite whiskers with a low surface energy and high aspect ratio are beneficial for enhancing the robustness of the superhydrophobic coating. Fig. $7(\mathrm{a}$ and $\mathrm{b})$ shows the morphology of the superhydrophobic coating and a cartoon of a water droplet deposited on the superhydrophobic coating surface, respectively. Anhydrite whiskers are randomly
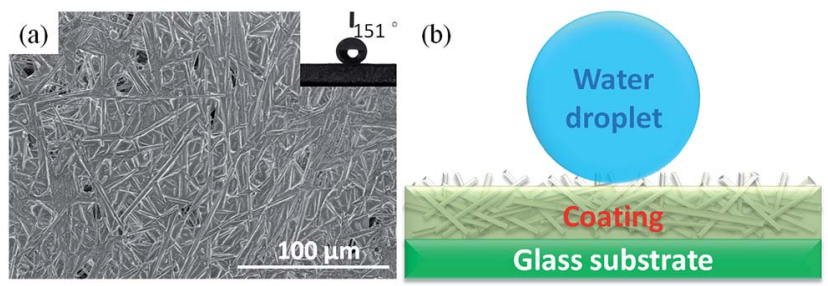

Fig. 7 (a) SEM image of the superhydrophobic coating surface, and (b) cartoon of a water droplet deposited on the superhydrophobic coating surface.

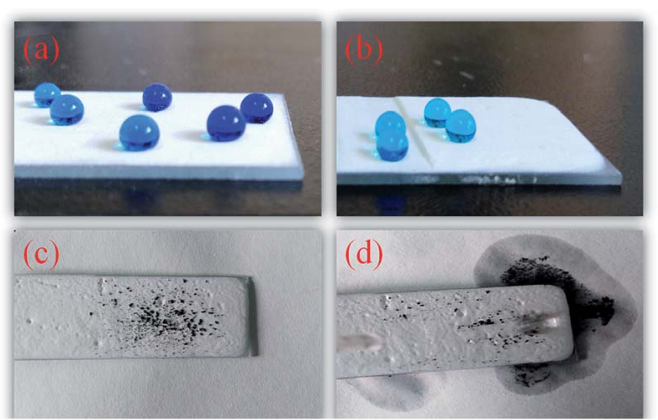

Fig. 8 Photographs of water droplets (dyed with methylene blue) on the coated glass substrate: (a) intact coating, and (b) coating is cut partly with a knife; ( $c$ and d) water droplet removing dirt from the coated glass substrate.

agglomerated creating pits and pores on the glass substrate surface. The air is trapped in the cavity. It can greatly decrease the solid-liquid interfacial interactions when the liquid droplets are in contact with the superhydrophobic coating surface (Fig. 7a). This roughness structure is similar to the Cassie-Baxter regime as described in the cartoon, promoting superhydrophobicity ${ }^{37}$ (Fig. 7b). The contact angle of the superhydrophobic coating reaches $151^{\circ}$.

The water repellency of the coating is highlighted in Fig. 8a, where water droplets exhibit typical spherical shapes on the coating surface. Importantly, as shown in Fig. 8b, the coating can be worn without sacrificing its original superhydrophobicity. It is important to extend the service lifespan of the superhydrophobic coating. The self-cleaning properties of superhydrophobic coatings are important for their practical applications. Fig. 8(c and d) shows the self-cleaning process of the superhydrophobic coating, which was photographed with a digital camera. The droplets roll down easily from the coating surface and take the contaminant away from the coating surface at the same time, keeping the coating surface clean. This result shows that the as-prepared coating can protect substrates from dust pollution in practical applications.

Fig. 9 shows the cross-cut tape adhesion test of the coated sample according to the ASTM D 3359 standard. From Fig. 9b, it can be seen that the coating shows lower removal of the coating from chipping around the cut lines and the cross hatch squares. The cross-cut damage of the coating is rated as $3 \mathrm{~B}$ according to
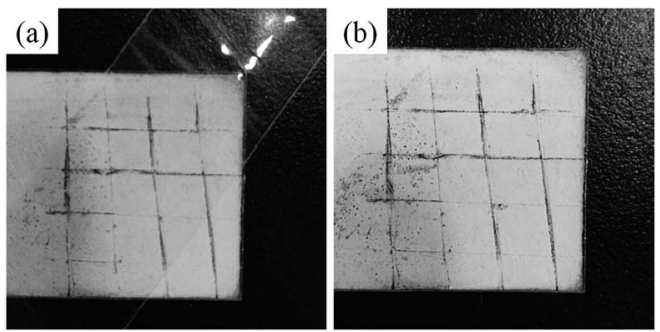

Fig. 9 Adhesion tests conducted on the coated sample according to ASTM D 3359: (a) pristine sample; (b) after the test. 
ASTM D 3359. The results indicate that the coating has good adhesion quality.

\section{Conclusions}

In summary, hydrophobic anhydrite whiskers are fabricated by TEOS hydrolysis and condensation and subsequent surface modification with OTS. The thickness of the surface modification coating layer is approximately $15 \mathrm{~nm}$. The contact angle of the anhydrite whiskers can reach up to $123^{\circ}$. The superhydrophobic coating is fabricated via a facile method using hydrophobic anhydrite whiskers. The superhydrophobic coatings show a high contact angle and a self-cleaning effect. The freshly exposed surface created by cutting or abrasion also exhibits superhydrophobicity. We believe that the findings in this study are important for designing durable and long service lifespan superhydrophobic coatings.

\section{Conflicts of interest}

The authors declare no competing financial interest.

\section{Acknowledgements}

We gratefully acknowledge projects from the Science and Technology project Foundation of Qinghai Province, China (grant number 2015-ZJ-737) and the National Natural Science Foundation of China (grant number 51402323).

\section{References}

1 P. Ragesh, V. A. Ganesh, S. V. Nair and A. S. Nair, J. Mater. Chem. A, 2014, 2, 14773-14797.

2 K. K. Tu, X. Q. Wang, L. Z. Kong, H. J. Chang and J. L. Liu, RSC Adv., 2016, 6, 701-707.

3 H. L. Yu, J. Q. Zhu, L. Yang, B. Dai, L. Baraban, G. Cuniberti and J. C. Han, Mater. Des., 2015, 87, 198-204.

4 S. Ramakrishna, K. S. Santhosh Kumar, D. Mathew and C. P. Reghunadhan Nair, Sci. Rep., 2015, 5, 18510.

5 J. Y. Huang, S. H. Li, M. Z. Ge, L. N. Wang, T. L. Xing, G. Q. Chen, X. F. Liu, S. S. Al-Deyab, K. Q. Zhang, T. Chen and Y. K. Lai, J. Mater. Chem. A, 2015, 3, 2825-2832.

6 L. Wu, J. P. Zhang, B. C. Li and A. Q. Wang, J. Mater. Chem. B, 2013, 1, 4756-4763.

7 J. X. Wu, J. Y. Li, Z. Q. Wang, M. Yu, H. Q. Jiang, L. F. Li and B. W. Zhang, RSC Adv., 2015, 5, 27752-27758.

8 L. Wang, Q. H. Gong, S. H. Zhan, L. Jiang and Y. M. Zheng, Adv. Mater., 2016, 28, 7729-7736.

9 A. Milionis, E. Loth and I. S. Bayer, Adv. Colloid Interface Sci., 2016, 229, 57-79.

10 L. Cao, Y. Wan and Q. Zhang, J. Non-Cryst. Solids, 2015, 410, 35-38.

11 T. Verho, C. Bower, P. Andrew, S. Franssila, O. Ikkala and R. H. A. Ras, Adv. Mater., 2011, 23, 673-678.
12 Y. Q. Wang, Y. C. Li, A. Yuan, B. Yuan, X. R. Lei, Q. Ma, J. Han, J. X. Wang and J. Y. Chen, Cryst. Res. Technol., 2014, 49, 800-807.

13 J. Y. Liu, L. Ren, Q. Wei, J. L. Wu, S. Liu, Y. J. Wang and G. Y. Li, eXPRESS Polym. Lett., 2011, 5, 742-752.

14 M. Miao, X. Feng, G. L. Wang, S. M. Cao, W. Shi and L. Y. Shi, Particuology, 2015, 19, 53-59.

15 Q. Han, K. B. Luo, H. P. Li and L. Xiang, Particuology, 2014, 17, 131-135.

16 W. J. Yuan, Y. H. Lu and S. A. Xu, Materials, 2016, 9, 625.

17 G. W. Zhang, F. Z. Wang, Z. X. Huang, J. Dai and M. X. Shi, Materials, 2016, 9, 723.

18 J. Y. Wang, H. B. Jia, J. J. Zhang, L. F. Ding, Y. Huang, D. P. Sun and X. D. Gong, J. Mater. Sci., 2014, 49, 6093-6101.

19 X. Feng, Y. Zhang, G. L. Wang, M. Miao and L. Y. Shi, Powder Technol., 2015, 271, 1-6.

20 T. C. Lee and J. X. Deng, Ceram. Int., 2001, 27, 755-760.

21 T. Z. Hong, Z. H. Lv, X. Liu, W. Li, X. Y. Nai and Y. P. Dong, Mater. Des., 2016, 107, 117-122.

22 X. Y. Liu, Y. H. Liu, J. H. Yang, L. L. Yang, J. Cao, X. W. Meng, D. L. Han, S. Yang, M. B. Wei and Y. Q. Liu, J. Mater. Sci., 2016, 27, 14-22.

23 J. Cao, B. J. Wang, D. L. Han, S. Yang, Q. Y. Liu, T. T. Wang, H. F. Niu and J. H. Yang, Mater. Lett., 2014, 135, 71-74.

24 C. L. Zhang, Z. H. Li, Q. H. Li, L. W. Han, J. L. Zhu, Y. L. Bai, C. Ge, Y. T. Zhao and H. B. Zhong, J. Nanosci. Nanotechnol., 2016, 16, 2277-2282.

25 X. He, Q. Yang, L. J. Fu and H. M. Yang, Funct. Mater. Lett., 2015, 8, 1550056.

26 P. H. Pi, C. Liu, X. F. Wen, L. F. Zheng, S. P. Xu and J. Cheng, Particuology, 2015, 19, 93-98.

27 B. Hui, Y. Y. Li, Q. T. Huang, G. L. Li, J. Li, L. P. Cai and H. P. Yu, Mater. Des., 2015, 84, 277-284.

28 Z. W. Zhu, G. H. Xu, Y. An and C. H. He, Colloids Surf., A, 2014, 457, 408-413.

29 L. Black, K. Garbev, P. Stemmermann, K. R. Hallam and G. C. Allen, Cem. Concr. Res., 2003, 33, 899-911.

30 L. Black, A. Stumm, K. Garbev, P. Stemmermann, K. R. Hallam and G. C. Allen, Cem. Concr. Res., 2003, 33, 1561-1565.

31 F. Bellmann, T. Sowoidnich, H.-M. Ludwig and D. Damidot, Cem. Concr. Res., 2012, 42, 1189-1198.

32 V. Rheinheimer and I. Casanova, Cem. Concr. Res., 2014, 60, 83-90.

33 L. Black, A. Stumm, K. Garbev, P. Stemmermann, K. R. Hallam and G. C. Allen, Cem. Concr. Res., 2005, 35, 51-55.

34 Y. L. Wang and M. Lieberman, Langmuir, 2003, 19, 11591167.

35 P. Balan, R. K. S. Raman, E.-S. Chan, M. K. Harun and V. Swamy, Prog. Org. Coat., 2016, 90, 222-234.

36 S. A. Mirji, Surf. Interface Anal., 2006, 38, 158-165.

37 B. C. Li and J. P. Zhang, Chem. Commun., 2016, 52, 2744. 